\title{
Comprimento focal de lentes esféricas
}

\author{
Diogo Soga*1, Raul Dias Paiva Jr. ${ }^{2}$, Mikiya Muramatsu ${ }^{1}$ \\ ${ }^{1}$ Instituto de Física, Universidade de São Paulo, Rua do Matão, 1371 prédio Ala 1, São Paulo, SP, Brazil \\ ${ }^{2}$ Centro Universitário SENAC, São Paulo, SP, Brazil
}

Recebido em 04 de Outubro, 2016. Revisado em 04 de Janeiro, 2017. Aceito em 06 de Fevereiro, 2017.

Uma lente esférica não se comporta como uma lente fina pois sua espessura não é desprezível. Neste trabalho apresentamos a dedução da equação do comprimento focal de uma lente esférica por meio da técnica de traçado de raios. Comparamos o resultado obtido com a expressão de comprimento focal de lentes grossas obtido pelo método matricial. Também aplicamos a expressão para o caso de uma esfera de água e comparamos com valores experimentais.

Palavras-chave: comprimento focal, lentes esféricas, esfera de água, lentes grossas.

A spherical lens can not be considered as a thin lens because the thickness is not negligible. In this work we present the deduction of the focal length equation for a spherical lens, developed through ray tracing technique. We compared the result with the focal length equation for thick lens obtained from matrix formalism. We also used the equation for a water sphere and compared with experimental data. Keywords: focal length, spherical lens, water sphere, thick lens.

\section{Introdução}

Existem iniciativas para melhorar o ensino, tal como a instrumentalização [1], que auxilia o educador a trazer a realidade mais perto do aluno, com a visualização dos fenômenos físicos e de suas aplicações.

$\mathrm{Na}$ Literatura encontramos trabalhos sobre a construção de microscópios ópticos caseiros 2 25, visando o Ensino de Ciências em sala de aula. Seguindo nesse tema, temos o trabalho de Vannoni e outros [3], que apresenta um modelo de microscópio cujo formato assemelha-se ao microscópio composto apresentado em alguns livros didáticos [6 8]. O microscópio proposto pode ser composto por duas lentes (lentes objetiva e ocular) ou três lentes (lentes objetiva, de campo e ocular), instalados em um tubo junto a um suporte vertical. Em formato diferente, o trabalho de Sepel e outros [4] apresenta um modelo de microscópio construído com um gargalo de garrafa plástica (PET), onde a rosca do gargalo funciona como um sistema para ajuste de foco da lente, que está fixada na tampa. Enquanto que, no

*Endereço de correspondência: diogosp@usp.br trabalho de Myint e outros [5], o modelo difere mais porque utiliza uma gota de água para atuar como uma lente objetiva, e o suporte dessa lente também tem formato diferenciado.

Alguns microscópios ópticos podem conter esferas de vidro que formam a imagem de objetos. Em uma esfera de vidro de raio $R$ atuando como uma lente, sua espessura $(2 R)$ é da mesma ordem de grandeza dos raios de curvatura da superfície da lente, tanto da interface de entrada $R_{1}$ quanto da interface de saída $R_{2}$. Então a espessura não é desprezível, como se considera na dedução da fórmula de Gauss [6], o que inviabiliza a utilização desta última. Algumas vezes a fórmula é chamada como "equação dos fabricantes de lentes" 9,10 , a qual é normalmente apresentada em livros textos do Ensino Médio 11. Uma expressão para o cálculo do comprimento focal dessas esferas de vidro pode ser encontrada no sítio da Optipedia [12, porém não há explicação sobre as hipóteses utilizadas nem as limitações dessa expressão. Apresentamos neste trabalho uma forma de dedução dessa expressão utilizando a técnica de traçado de raios 6 . Comparamos com outros traba- 
lhos encontrados na Literatura e apresentamos uma aplicação.

\section{Comprimento focal de uma esfera}

A dedução da expressão para o cálculo do comprimento focal inicia-se considerando uma esfera uniforme (figura 1), de índice de refração $n$ e raio $R$, cujo centro é o ponto $C$, e por este ponto passa uma reta que chamamos de eixo óptico. Esta esfera é iluminada por um raio de luz que incide na interface do lado esquerdo e sai pela interface do lado direito. O raio de luz incide na superfície da lente a um ângulo $\alpha$ em relação a normal da superfície, mas paralelo ao eixo óptico, a uma altura $y$ em relação ao eixo óptico. Após refratar na primeira superfície da lente, o raio segue com ângulo $\beta$ em relação a normal da superfície, e com ângulo $\delta$ em relação a reta paralela ao eixo óptico. Depois o raio de luz incide na segunda interface a um ângulo $\gamma$ em relação à normal, e com ângulo $\delta$ em relação a outra reta paralela ao eixo óptico e a altura $x$ em relação ao eixo óptico. Neste ponto o raio de luz refrata para o meio externo a um ângulo $\varepsilon$ em relação à normal à superfície e a um ângulo $\varphi$ em relação à reta paralela ao eixo óptico. A distância entre o centro $C$ (que na aproximação paraxial que utilizaremos está contido no plano principal da lente [6]) e o ponto de convergência do raio de luz com o eixo óptico é chamada distância focal $f$. Enquanto que a distância entre o ponto de intersecção entre a interface da esfera e o eixo óptico até o ponto de convergência do raio de luz com o eixo óptico é chamada de distância focal

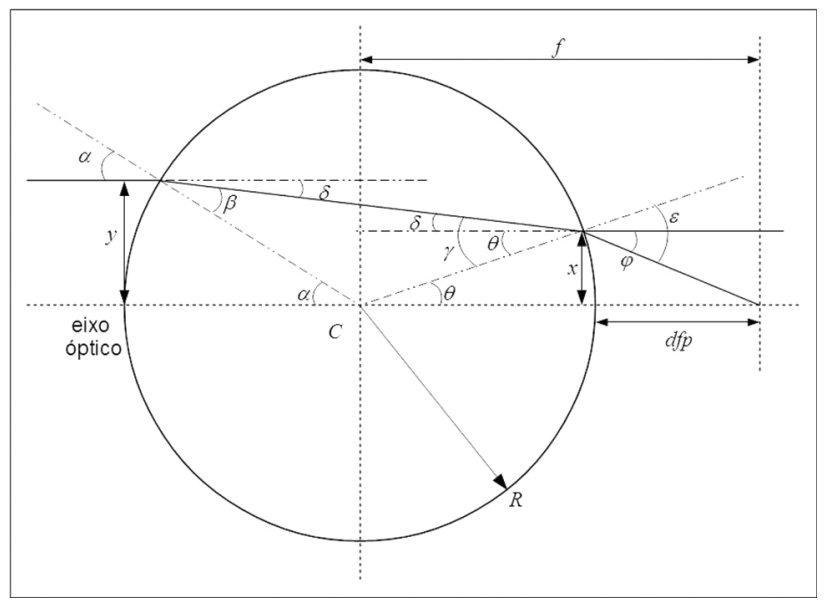

Figura 1: Esquema de traçado de raios em uma lente esférica. posterior $(d f p)[6]$.

Inicialmente, consideramos a aproximação paraxial $y \ll R$, então o ângulo $\alpha$ é pequeno e podemos aproximar:

$$
\sin (\alpha)=\frac{y}{R} \approx \alpha .
$$

Procedendo da mesma maneira para o ângulo $\beta$ :

$$
\sin (\beta) \approx \beta
$$

Sendo o meio externo o ar, $n_{\text {ar }}=1, n>1$ (vidros comuns tem valores típicos de 1,5 ) e usando a Lei de Snell [6], temos no ponto de entrada da lente:

$$
n_{\text {ar }} \sin (\alpha)=n \sin (\beta),
$$

das expressões 1 e 2 obtemos:

$$
\frac{y}{R}=n \beta \Rightarrow \beta=\frac{y}{n R} .
$$

$\mathrm{Na}$ interface de entrada, temos a relação entre ângulos:

$$
\alpha=\delta+\beta \Rightarrow \delta=\alpha-\beta,
$$

pelas equações 1 e 4 obtemos:

$$
\delta=\frac{y}{R}\left(\frac{n-1}{n}\right) .
$$

Na figura 1. temos a relação entre as alturas $x$ e $y:$

$$
x=y-2 R \tan (\delta),
$$

considerando $\delta<\alpha: \sin (\delta) \approx \delta$ e de modo similar $\tan (\delta) \approx \delta$, então:

$$
x=y-2 R \delta,
$$

substituindo $\delta$ da equação 6 , temos:

$$
x=y\left(\frac{2-n}{n}\right) \text {. }
$$

Na interface de saída, o ângulo $\theta$ é dado por:

$$
\theta \approx \frac{x}{R}
$$

e a relação entre os ângulos é dada por:

$$
\gamma=\theta+\delta=\frac{y}{R}\left(\frac{2-n}{n}\right)+\frac{y}{R}\left(\frac{n-1}{n}\right)=\frac{\alpha}{n},
$$


onde foram utilizadas as expressões 9, 6 e 1 .

Agora aplicando a Lei de Snell na interface de saída da lente:

$$
n \sin (\gamma)=n_{a r} \sin (\varepsilon),
$$

usando as aproximações $\sin (\gamma) \approx \gamma \mathrm{e} \sin (\varepsilon) \approx \varepsilon$, e substituindo $\gamma$ pela expressão 11, obtemos:

$$
\varepsilon=\alpha .
$$

No lado externo da esfera, temos a seguinte relação entre os ângulos $\varphi, \varepsilon$ e $\theta$ dada por:

$$
\varphi=\varepsilon-\theta,
$$

usando as expressões 13, 10, 9 e 1, obtemos:

$$
\varphi=\alpha-\frac{x}{R}=\frac{2 y(n-1)}{R n} .
$$

O ângulo $\varphi$ (figura 1) também pode ser calculado fazendo a seguinte aproximação:

$$
\tan (\varphi) \approx \frac{y}{f} \approx \varphi
$$

das expressões 15] e 16, obtemos a expressão do comprimento focal $f$ :

$$
f=\frac{R n}{2(n-1)} .
$$

O resultado acima foi obtido considerando que a lente está imersa no ar, $n>1$, que os raios de luz que atravessam a lente sejam apenas os raios próximos ao eixo óptico da lente e utilizamos a Lei de Snell nas interfaces da lente. A partir de $f$, obtemos $d f p$ por:

$$
d f p=f-R
$$

\section{Lentes espessas}

Na Literatura encontramos uma expressão para o cálculo do comprimento focal $f$ de uma lente espessa [6, 13 imersa no ar:

$$
\frac{1}{f}=(n-1)\left(\frac{1}{R_{1}}-\frac{1}{R_{2}}\right)+\frac{(n-1)^{2}}{n}\left(\frac{t}{R_{1} R_{2}}\right),
$$

onde $R_{1}$ é o raio de curvatura da superfície a esquerda, $R_{2}$ é o raio de curvatura da superfície a direita, $t$ é a espessura da lente e $n$ é o índice de refração da lente. Essa expressão foi deduzida por meio do método matricial [6, onde cada elemento óptico é representado por uma matriz, e um raio de luz é transformado pela matriz que representa a lente. Para uma lente fina o valor de $t$ é desprezível, então o segundo termo é zero e resta apenas o primeiro termo, que é igual a fórmula de Gauss.

Aplicando ao caso de uma lente esférica, onde $R_{1}=R, R_{2}=-R$, e espessura $t=2 R$, obtemos:

$$
\frac{1}{f}=(n-1)\left(\frac{1}{R}+\frac{1}{R}\right)+\frac{(n-1)^{2}}{n}\left(\frac{-2 R}{R R}\right),
$$

logo:

$$
f=\frac{n R}{2(n-1)}
$$

que é igual a expressão que foi deduzida acima na equação 17, confirmando a relação entre $f$ e $R$.

Cabe ressaltar que Helene e Helene [14 construíram um modelo de olho humano com uma esfera de vidro, e mostraram que a expressão do comprimento focal desse modelo é:

$$
f=\frac{n R}{(n-1)} \text {. }
$$

Esta expressão também foi deduzida pelo traçado de raios, mas difere da equação 17 pela ausência do fator dois no divisor. Essa diferença é justificada porque, para o modelo do olho humano, foi considerada apenas a refração na superfície de entrada da esfera, uma vez que os autores estavam interessados na projeção da imagem na segunda superfície da esfera, como ocorre no olho humano, onde a imagem é projetada sobre a retina.

Pelo exposto acima, vemos a importância da equação 17. pois a equação de Gauss não é adequada quando temos lentes espessas.

\section{Esfera de água}

Para ilustrar a utilidade da equação 17, apresentamos a medida de $d f p$ de uma bolha de vidro (figura 2), cheia de água destilada e lacrada. Considerando as paredes da bolha tão finas que podemos desprezar os seus efeitos, vamos considerá-la como uma esfera de água. Suspendemos a esfera sobre uma mesa e usamos ela para formar a imagem de uma luminária (figura 3), com quatro lâmpadas fluorescentes e está acima da esfera a cerca de $2 \mathrm{~m}$ de altura (assim podemos considerar que os feixes de luz vem paralelos 


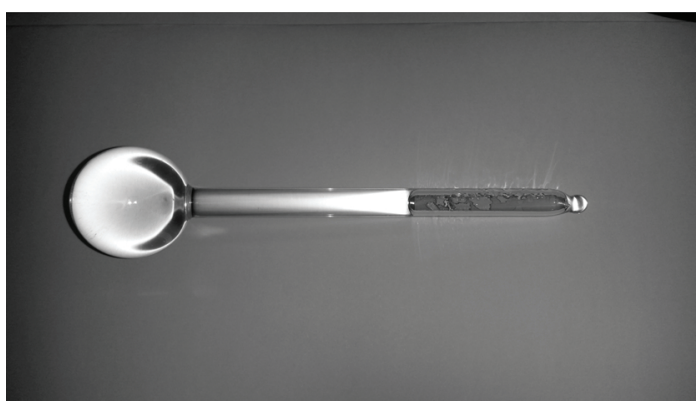

Figura 2: Bolha de vidro cheia de água destilada e lacrada.

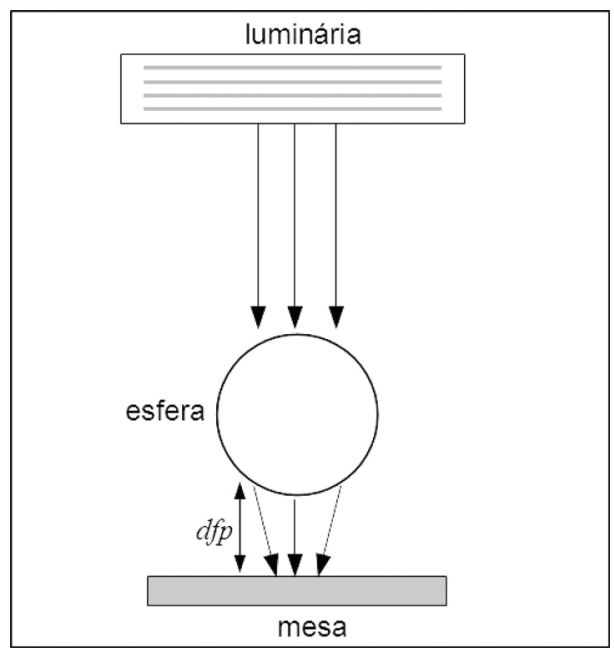

Figura 3: Esquema de formação de imagem da luminária sobre a mesa.

entre si). Projetamos a imagem da luminária em um papel sobre a mesa (figura 4). O raio da esfera é $(13,15 \pm 0,05) \mathrm{mm}$, o índice de refração $n$ da água é $1,333[6], \operatorname{logo}: f=(26,6 \pm 0,1) \mathrm{mm}$. Subtraindo o raio da esfera, o valor de $d f p$ é $(13,2 \pm 0,1) \mathrm{mm}$.

Devido ao excesso de luminosidade da luminária, tivemos dificuldade para ajustar a distância focal com precisão, então medimos as distâncias anterior e posterior ao ponto focal onde percebemos a distorção da imagem. A distância focal estará entre esses dois valores. Calculando o valor médio dessas distâncias, obtemos o valor de $d f p$ e o resultado é $(13 \pm 1) \mathrm{mm}$. Este valor é compatível com o valor calculado pela expressão 17

Para montar uma esfera de água, sugerimos usar uma lâmpada de filamento e de vidro transparente, retirando a base e o filamento 15, depois preencher com água e vedar. O bulbo das lâmpadas de geladeira tem o formato mais esférico que outros tipos de lâmpadas, tornando-as mais indicadas para este experimento.

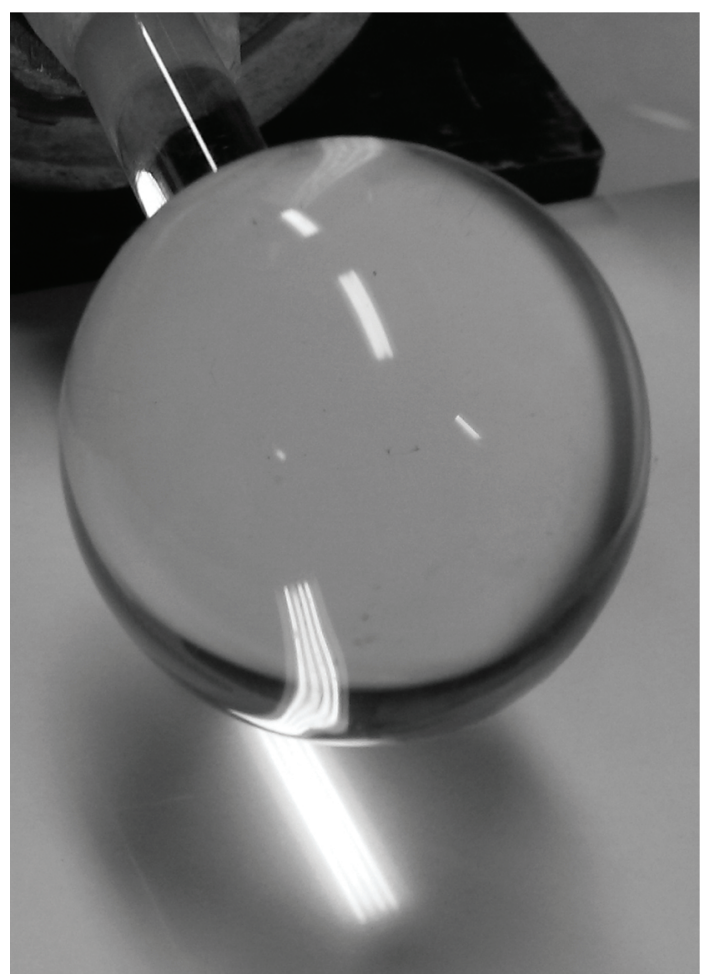

Figura 4: Esfera de água formando uma imagem na mesa.

\section{Conclusão}

Apresentamos a dedução do comprimento focal $f$ de uma esfera imersa no ar, por meio da técnica de traçado de raios, onde consideramos apenas os raios paraxiais. Foram utilizadas aproximações de algumas funções trigonométricas. A expressão deduzida é compatível com expressões de comprimento focal de lentes grossas encontradas na Literatura. Com isto, fica evidente a limitação da fórmula de Gauss, ensinada nos cursos de Óptica. Medimos o valor de $d f p$ de uma esfera de água, com resultados compatíveis com o obtido pela expressão deduzida. Esse experimento pode ser implementado como uma atividade prática em cursos de Física básica, devido a sua simplicidade, e a dedução da expressão é um excelente exemplo da aplicação da Lei de Snell, com conteúdo de Trigonometria.

\section{Agradecimentos}

Os autores agradecem o auxílio técnico de Claudio Hiroyuki Furukawa (Instituto de Física da USP), pela confecção da esfera de água utilizada neste trabalho. Também agradecem a Sarah Isabel P.M.N. Alves, Isis V. de Brito e Michele H. Ueno-Guimarães, pelas sugestões na elaboração do trabalho. 


\section{Referências}

[1] W.L. Almeida, F.M.M. Luz, J.B. Silva, S.L.R. Silva e A.M. Brinatti, Caderno Brasileiro Ensino de Física 30, 396 (2013).

[2] PIBID UFSM CAPES, Construindo Microscópios Reciclados, disponível em http://www.youtube. com/watch?v=7-PwVOkfaKM, acesso em 23/4/2014.

[3] M. Vannoni, P.K. Buah-Bassuah and G. Molesini, Physics Education 42, 385 (2007).

[4] L.M.N. Sepel, J.B.T. Rocha and E.L.S. Loreto, Genética na Escola 6, 1 (2011).

[5] H.H. Myint, A.M. Marpaung, H. Kurniawan, H. Hattori and K. Kagawa, Physics Education 36, 97 (2001).

[6] E. Hecht, Óptica (Fundação Calouste Gulbenkian, Lisboa, 1991).

[7] H.D. Young, Física IV : Ótica e Física Moderna / Young e Freedman (Pearson Education do Brasil, São Paulo, 2009).

[8] J.D. Cutnell e K.W. Johnson, Fúsica v. 2 (Editora LTC, Rio de Janeiro, 2006).

[9] R.A. Serway e J.W. Jewett Jr. Princípios de Física - Volume 4: Óptica e Física Moderna (Cengage Learning, São Paulo, 2005).

[10] H.D. Young e R.A. Freedman, Sears $\&$ Zemansky Fúsica IV Ótica e Fúsica Moderna (Pearson Education do Brasil, São Paulo, 2011).

[11] A. Gaspar, Física - Ondas, óptica, Termodinâmica (Editora Ática, São Paulo, 2000).

[12] OPTIPEDIA, Ball Lens, disponível em http://spie.org/x34513.xml?pf=true, acesso em $30 / 5 / 2014$.

[13] N. Carlin, E.M. Szanto, F.O. Jorge, F.A. Souza, L.H. Bechtold e W.A. Seale, Revista Brasileira de Ensino de Física 29, 299 (2007).

[14] O. Helene e A.F. Helene, Revista Brasileira de Ensino de Física 33, 3312 (2011).

[15] Manual do Mundo, Como Fazer Fogo com Água (Experimento de Física), disponível em https:// wWw youtube.com/watch?v=BprWYzk-AMI, acesso em $26 / 4 / 2016$. 